\title{
Semantic predictability eliminates the transposed-letter effect
}

\author{
Steven G. Luke • Kiel Christianson
}

Published online: 13 December 2011

(C) Psychonomic Society, Inc. 2011

\begin{abstract}
Semantic predictability facilitates word recognition during language processing. One possible explanation for this facilitation is that highly specific predictions generated online during language processing preactivate some features of upcoming words. To explore whether, how, and when these predictions affect visual word recognition, in the two experiments reported here we investigated the influence of semantic predictability on transposed-letter priming. In order to do so, a paradigm that combines self-paced wordby-word reading with masked priming was developed. Transposed-letter priming occurred in nonconstraining contexts but not in constraining contexts, indicating that readers use context to make predictions about both letter identity and position in upcoming words, and that these predictions have an early influence on visual word recognition.
\end{abstract}

Keywords Predictability P Prediction · Transposed-letter priming $\cdot$ Masked priming $\cdot$ Reading $\cdot$ Visual word recognition $\cdot$ Lexical processing $\cdot$ Psycholinguistics

Words that appear in sentence contexts can sometimes be highly predictable. For example, most readers who see the partial sentence "I can't breathe because my tie is too ..." would expect that the next word would be tight. Semantic predictability of this sort is known to have a significant

S. G. Luke $(\square)$

Department of Psychology, University of South Carolina,

1512 Pendleton Street,

Columbia, SC 29208, USA

e-mail: lukesg@mailbox.sc.edu

\section{K. Christianson}

Department of Educational Psychology and Beckman Institute for Advanced Science and Technology, University of Illinois, Urbana-Champaign, IL, USA influence on word recognition, speeding response times in lexical decision and naming tasks (Duffy, Henderson, \& Morris, 1989; Fischler \& Bloom, 1979; Hess, Foss, \& Carroll, 1995; Stanovich \& West, 1979). Predictability also affects eye movements during reading (Ehrlich \& Rayner, 1981; Kliegl, Grabner, Rolfs, \& Engbert, 2004; Morris, 1994; Rayner \& Well, 1996; Zola, 1984). Rayner and Well's findings are typical of the eyetracking literature: They observed decreased fixation times for medium- and high-constraint target words as compared to low-constraint words. Additionally, they observed increased skipping of high-constraint (but not medium-constraint) target words, although fixation probability was still high (.78) for the high-constraint words. A great deal of electrophysiological evidence has also accumulated regarding the effect of semantic predictability from studies investigating eventrelated potentials (ERPs; Federmeier \& Kutas, 1999; Kutas \& Hillyard, 1984; van Berkum, Brown, \& Hagoort, 1999). For example, Kutas and Hillyard presented participants with sentences that ended in words with varying degrees of predictability and observed that N400 responses decreased for more predictable words (i.e., words with higher cloze probability).

Clearly, semantic predictability influences response times, eye movements, and electrophysiological responses, although how this influence is exerted is a subject of some debate. There are two main ideas about how predictability might increase processing efficiency. Many researchers have suggested that the influence of predictability arises after lexical access, with predictability making it easier to semantically integrate the word into the rest of the sentence (Forster, 1989; Marslen-Wilson, 1989; Norris, 1994; Stanovich \& West, 1983). Other researchers have claimed that readers (and listeners) actively predict the identity of the upcoming word, so that predictability effects occur 
during lexical access (Federmeier, 2007; Lau, Almeida, Hines, \& Poeppel, 2009). Evidence for the second position comes primarily from the ERP literature, for instance from Federmeier and Kutas (1999), who observed smaller N400s to pines than to tulips as a completion for "They wanted to make the hotel look more like a tropical resort. So, along the driveway, they planted rows of . . .," where palms is the expected completion. They concluded that semantic predictability facilitates word recognition by preactivating semantic features, which is why the greater semantic overlap between pines and the expected word palms led to a smaller N400 than did tulips. There is some eyetracking evidence for prediction as well. Using the visual-world paradigm, Altmann and Kamide (1999; Kamide, Altmann, \& Haywood, 2003) observed that participants used verb information to restrict the domain of possible target objects and moved their eyes to a highly predictable target object even before that object was named.

The ERP literature suggests not only that predictions are made, but that these predictions can be quite specific, even down to the levels of morphosyntax and phonology (DeLong, Urbach, \& Kutas, 2005; Otten, Nieuwland, \& van Berkum, 2007; van Berkum, Brown, Zwitserlood, Kooijman, \& Hagoort, 2005; Wicha, Bates, Moreno, \& Kutas, 2003; Wicha, Moreno, \& Kutas, 2003, 2004). For example, DeLong et al. presented participants with sentences such as "The day was breezy so the boy went outside to fly a/an ...," where the most likely continuation is the word kite, which requires $a$ and not $a n$ as its indefinite article. They observed increased N400 responses to indefinite articles that did not match the phonology of the expected (but not yet visible) word, indicating that participants had predicted not just the semantic content of the upcoming word, but its phonological content as well.

Laszlo and Federmeier (2009) presented evidence that readers' predictions can also contain orthographic information. Laszlo and Federmeier measured ERPs as people read sentences ending in a highly predictable word, an orthographic neighbor of the highly predictable word, or an orthographically unrelated word. They observed decreased surprise as indicated by reduced N400 waveforms in response to orthographic neighbors of the expected words as compared to unrelated words. They interpreted this result as evidence of "a considerable degree of specificity in predictive processing" (p. 334), down to the level of orthographic representation.

The observation that readers predict even the individual letters and sounds within a word is consistent with the observation that predictability effects arise quite early in reading (Frisson, Rayner, \& Pickering, 2005; Morris, 1994). On the basis of this evidence, advocates of active prediction have contended that semantic predictability has its influence during lexical access. Fortunately, the process of lexical access has been studied extensively, so the influence of predictability on lexical access during reading can be investigated using the theories and findings of the visual word recognition literature as a guide. Once such finding, called the transposed-letter (TL) effect, has the potential to reveal a great deal about how and when semantic predictability influences word recognition. The TL effect is based on a rapidly growing body of research in visual word recognition that has demonstrated that under most circumstances, letter transpositions are significantly less disruptive to word recognition than appropriately matched letter substitutions (Andrews, 1996; Chambers, 1979; Forster, Davis, Schoknecht, \& Carter, 1987; Holmes \& Ng, 1993; O’Connor \& Forster, 1981; Perea \& Lupker, 2003a, 2003b, 2004; Schoonbaert \& Grainger, 2004). The TL effect suggests a dissociation between letter identity and letter position encoding and reveals that letters are not strictly bound to particular positions, at least at the early stages of visual word recognition.

Typically, the TL effect has been observed with letter transpositions in masked-priming lexical-decision tasks, in which the primes are presented for very brief durations, typically $30-60 \mathrm{~ms}$, and masked so that they are not consciously visible to participants (Forster, Mohan, \& Hector, 2003; Perea \& Lupker, 2003a, 2003b, 2004). The primes are constructed so as to create nonwords, and the target word is the correctly spelled word. For example, if the target of a lexical decision task is JUDGE, primes with internal transpositions, such as jugde, typically result in responses that are faster than those following primes with letter substitutions, such as jupbe, and sometimes that are not significantly slower than responses following correctly spelled primes (e.g., judge). This effect also holds when transposed letters are nonadjacent (e.g., caniso vs. camico when the target is CASINO; Perea \& Lupker, 2004), as well as in masked-priming naming tasks (Christianson, Johnson, \& Rayner, 2005). However, letter transpositions become disruptive for unmasked stimuli (White, Johnson, Liversedge, \& Rayner, 2008), suggesting that letter position is only flexible in the earlier stages of visual word recognition.

The observation that letter transpositions are far less disruptive than are matched letter substitutions has proved critical in developing and testing models of visual word recognition (cf. Gomez, Ratcliff, \& Perea, 2008; Grainger \& Whitney, 2004; Lupker, Perea, \& Davis, 2008; Perea \& Lupker, 2003a, 2003b, 2004). It has also led to a small revolution in the application of the transposed-letter effect to explore a variety of phenomena, including neighborhood effects on visual word recognition (Acha \& Perea, 2008a) and visual word recognition in more or less transparent orthographies (Ktori \& Pitchford, 2008; Perea \& Carreiras, 2008), in developing readers (Acha \& Perea, 2008b), and in 
parafoveal processing in reading connected text (Johnson, Perea, \& Rayner, 2007). Various position-coding schemes have been proposed that take into account the observed flexibility when mapping letters onto positions. These models include SOLAR (Davis, 1999) and, more recently, the spatial-coding model (Davis, 2010), SERIOL (Whitney, 2001a, 2001b), the overlap model (Gomez et al., 2008), and the Bayesian reader model (Norris, Kinoshita, \& van Casteren, 2010)

There are limits on letter transposability, however, and these limits have also informed models of visual word recognition. The most frequently replicated finding in this regard is that transposing the beginning and ending letters is often more disruptive than internal transpositions (Chambers, 1979; Holmes \& Ng, 1993; Johnson et al., 2007; Perea \& Lupker, 2003a, 2003b; Rayner, White, Johnson, \& Liversedge, 2006). For example, Perea and Lupker (2003a) obtained a significant form-priming effect of $30 \mathrm{~ms}$ for word-internal transpositions (e.g., uhser $\rightarrow$ usher, as compared to ufner $\rightarrow$ usher), which was greater than the priming effect for word-final transpositions (e.g., ushre $\rightarrow$ usher, as compared to ushno $\rightarrow$ usher). These findings suggest that exterior letters are less "transposable" than interior ones.

The common thread uniting the models outlined above is that they are strongly bottom-up: Each model attributes both the TL effect and the observed limits on letter transposability to some property of the stimulus and allows little role for possible top-down influences such as lexical knowledge or predictions generated from context. Thus far, there has been little reason to criticize the exclusion of top-down factors from models of visual word recognition, but a small body of literature has suggested that certain top-down influences might modulate the effectiveness of TL primes. For example, such primes are not as effective when the primes themselves are words (e.g., clam does not prime calm; Duñabeitia, Perea, \& Carreiras, 2009; Gomez et al., 2008). Also, it has recently been observed that TL primes are not as facilitative to word recognition if the transpositions occur across morpheme boundaries (Christianson et al., 2005; Duñabeitia, Perea, \& Carreiras, 2007; but cf. Rueckl \& Rimzhim, 2011). There is no perceptual boundary between the last letter of one morpheme and the first letter of the next in the languages used in these studies; both within- and acrossmorpheme transpositions involved the transposition of two word-internal letters. Any disruption in the effectiveness of TL primes at a morpheme boundary could be argued to be the result of the application of prior knowledge - specifically, knowledge of which letter strings constitute legal morphemes. It has also been observed that orthotactic and phonotactic properties of the orthography and/or syllable structure of the language under investigation can modulate the effects of letter transposition (Lee \& Taft, 2009; Perea \& Carreiras, 2008).
All of the influences listed above-lexical status, morphology, and orthotactic and phonotactic constraints-are top-down, in the sense that they do not exist in the input, but rather represent some knowledge in the mind of the reader. Still, these constraints on the TL effect are all lexical in nature and can be applied to the current word without any consideration of the context in which the word appears, so in that way they differ significantly from semantic predictability. Thus, it is not known whether semantic predictability can influence letter transposability. Nevertheless, given the indications that not all letters are equally transposable, and that some top-down linguistic information influences the flexibility of letter position encoding, it seems prudent to test the possible influence of semantic predictability on the TL effect, and this has not, as yet, been tested empirically.

Although the effects of lexical predictability on eye movements during reading have been described in detail (e.g., Ehrlich \& Rayner, 1981; Rayner \& Well, 1996), to our knowledge no previous research has directly addressed predictability effects on the TL phenomena. The ERP evidence cited above indicates that readers make predictions about upcoming words that can include letter identity information, but since research on the TL effect reveals that letter identity and letter position encoding are separable, readers will not necessarily also predict the location of individual letters within a word. If letter position coding remains flexible in highly predictable words during reading (i.e., if the TL effect remains), this would suggest that semantic predictability has no influence on letter position encoding. On the other hand, if letter position coding becomes less flexible in highly predictable words (i.e., if the TL effect goes away), this would be consistent with the idea that readers make predictions during reading, and that a highly constraining context allows them to increase the specificity of those predictions, even allowing them to predict the identity and position of letters in an upcoming word. The former outcome would suggest that the influence of semantic predictability arises later in the word recognition process, while the latter outcome would suggest that this influence can be felt from the earliest moments of visual word recognition.

In sum, if it could be shown that semantic predictability can eliminate the effectiveness of masked TL primes, such a finding would provide behavioral evidence that semantic predictability leads readers to make highly specific orthographic predictions about upcoming words, including both letter identity and letter position. The purpose of the research reported here was to test the effect of semantic predictability on the effectiveness of masked TL primes. We did so by presenting the same set of TL prime-target pairs in nonconstraining sentence contexts, in Experiment 1, and in highly constraining contexts, in Experiment 2. 


\section{Experiment 1}

In order to explore top-down influences on the effectiveness of TL masked primes, it was first necessary to create a new tool that would combine the masked-priming paradigm with the presentation of entire sentences rather than isolated words. To do so, we have developed a paradigm that we call SPaM, because it combines self-paced word-by-word reading (Aaronson \& Ferres, 1984; Aaronson \& Scarborough, 1976; Just, Carpenter, \& Woolley, 1982) with masked priming. This paradigm is similar to one first described by Trueswell and Kim (1998), but it improves and expands on their paradigm in several ways (see the General Discussion). In Experiment 1, we tested the SPaM paradigm by using the exact items that had been used previously in an eyetracking study by Johnson et al. (2007), who used the boundary-change paradigm to present parafoveal previews of the TL versions of target words. These sentences were normed by Johnson et al. to verify that the fiveletter target words were not predictable from context. The target words and primes used in Johnson et al.'s sentences were, in turn, taken from Perea and Lupker (2003b), who had used the same items in a masked-priming lexical decision experiment. One key difference between these two studies was that Johnson et al. found that TL internal (e.g., jugde) and TL final (e.g., judeg) primes were equally facilitative when viewed in the parafovea, whereas Perea and Lupker (2003b) found (with foveal presentation) that only TL-internal primes produced unambiguous facilitation. Using these items in Experiment 1 allowed us to accomplish three goals. First, by comparing our results to those of Perea and Lupker (2003b), we were able to confirm that our synthesis of masked priming and self-paced reading produced priming that was comparable to the priming observed in a traditional masked-priming study. Second, given the evidence that even nonconstraining sentence contexts can influence the perceptual processing of words (Jordan \& Thomas, 2002), it was possible that simply embedding the prime-target pairs in any sentence would change the way that TL effects manifested, a possibility that Experiment 1 explored. Third, the new paradigm enabled us to confirm that the difference in priming patterns between the Perea and Lupker (2003b) and the Johnson et al. studies was attributable to parafoveal-versusfoveal processing differences, as Johnson et al. had speculated, rather than to some other difference between reading connected text and identifying individual words. Because in the new paradigm we presented the primes foveally, we expected the paradigm to yield the same pattern observed by Perea and Lupker (2003b) - namely, word-internal but not word-final TL priming effects - and this was exactly what we observed.

Method

Participants A group of 24 people from the University of Illinois at Urbana-Champaign community participated, the large majority of whom were recruited from the Educational Psychology subject pool. All were compensated for their time with either course credit or $\$ 5$.

Materials One hundred twenty sentences taken from Johnson et al. (2007, Exp. 1) were used in this experiment. Each sentence contained one five-letter target word. These sentences had been normed by Johnson et al. to verify that the five-letter target words were not predictable from the context. As we mentioned above, these words were the same ones used in Perea and Lupker's (2003b, Exp. 1) masked priming study. The frequency and other relevant characteristics of these words can be found in Perea and Lupker (2003b). The same six prime conditions used in Perea and Lupker (2003b) were reused here (see Table 1). The six prime conditions were (1) identity prime (the prime was identical to the target), (2) TL internal prime (two adjacent internal letters of the target were transposed), (3) substitution (SUB) internal prime (the two transposed internal letters of the TL internal prime were substituted with other letters of a similar overall shape), (4) TL final prime (the two final letters of the target were transposed), (5) SUB final prime (the two final letters of the TL final prime were substituted with other letters), and (6) unrelated word prime of the same length as the target. These six conditions were counterbalanced across six presentation lists in a Latin square design. The sentences were presented in a random order for each participant. Additionally, 40 yes/no comprehension questions were created for 40 of the target sentences. These questions were presented pseudorandomly to ensure that participants were paying attention to the task.

Procedure The experiment was run using E-Prime Professional software (Schneider, Eschman, \& Zuccolotto, 2002), version 2.0. Participants were seated $75 \mathrm{~cm}$ away from a 20in. monitor, with the refresh rate set to $100 \mathrm{MHz}$. Participants made responses on a standard game controller. All of the items were presented in black, 14-point text on a white background. The steps of the SPaM procedure described below are illustrated in Fig. 1. Participants first saw a fixation cross on the left side of the screen. When he or

Table 1 Example sentence with the six prime conditions in Experiment 1 Megan took the gray scarf out of the washer and put it in the dryer.

\begin{tabular}{ll}
\hline Identity & scarf \\
TL internal & scraf \\
SUB internal & scnef \\
TL final & scafr \\
SUB final & scatn \\
Unrelated & optic \\
\hline
\end{tabular}

TL, transposed letters; SUB, substituted letters. 


$$
\begin{aligned}
& \text { 1. I \#\#\# \#\#\#\#. (until button press) } \\
& \text { 2. - \#\#\# \#\#\#\#. (10 ms) } \\
& \text { 3. - buy \#\#\#\#. (50 ms prime) } \\
& \text { 4. - eat \#\#\#\#. (until button press) } \\
& \text { 5. - — \#\#\#\#. (10 ms) } \\
& \text { 6. __ spam. (50 ms prime) } \\
& \text { 7. _ _ spam. (until button press) }
\end{aligned}
$$

Fig. 1 The SPaM procedure

she pressed the NEXT button, the target sentence appeared on the screen, with hash marks (\#) in the place of letters. Each time the participant pressed the NEXT button, the following steps occurred. First, the letters of the currently visible word (word $N-1$ ) were replaced with underlines $\left(\_\right.$). Then, after a $10-\mathrm{ms}$ pause to ensure that the participant had completed a saccade to the next word (word $N$ ), the hash marks of word $N$ were replaced by the prime, which remained on screen for $50 \mathrm{~ms}$ (typical TL experiments use prime durations between 40 and $60 \mathrm{~ms}$ ). Unless word $N$ was a target word, the prime was the same word as word $N$. If word $N$ was a target, the prime was from one of the six conditions described above. After $50 \mathrm{~ms}$, the prime was replaced by the target word. A subsequent buttonpress replaced word $N$ with underlines, and after a 10-ms pause, the sequence began again for word $N+1$. Response times were measured from the onset of the target word. After the last word in the sentence was presented and the NEXT button was pressed, participants saw either a fixation cross again ( $2 / 3$ of the time) or a yes/no comprehension question in the center of the screen ( $1 / 3$ of the time); they answered the question by means of buttons labeled YES and NO on the game controller. Between each pair of sentences with questions, from zero to three intervening sentences appeared without questions. After the question was answered, the fixation cross reappeared and the process began again. The sentences were presented in a random order for each participant.

In masked priming, it is a common practice to present the prime in lowercase letters and the target in uppercase. This minimizes the overlap of visual features, which is important if priming is to be attributed to shared lexical representations, rather than, say, visual sensory memory. This practice was impractical here, as it would have required presenting the entire sentence in uppercase. Instead, the visual overlap of the prime and target was minimized by presenting them in different fonts and by italicizing the prime. The fonts used were Courier New, for the prime, and Lucida Console, for the target. Both fonts are monospace, meaning that all letters are the same width, which ensured that all primes and targets were the same width. With nonmonospace fonts, the primes and targets would not have appeared in the same location on the screen, which would have put the prime in a location other than where the participant was looking.

Previous integrations of masked priming with self-paced reading (Trueswell \& Kim, 1998) and with eyetracking (Sereno \& Rayner, 1992) had primed only the target word. Both of those studies had reported that participants noticed the prime quite often - so much so that it was necessary to instruct the participants to ignore the primes. Pilot testing of the SPaM method described here confirmed that when only one word in the sentence was primed, the presentation of the prime produced an obvious flash. To avoid this problem, we chose to prime every word, not just the target (primes for nontarget words were always identity primes). When every word was preceded by a prime, and therefore a flash, participants seldom if ever notice the presence of primes, so there was no need to draw attention to the prime by instructing participants to ignore it.

\section{Results and discussion}

Response times were measured from the onset of the target word, not from the previous buttonpress or the onset of the prime. Because the onsets of the prime, of the $10-\mathrm{ms} \mathrm{sac}-$ cade pause, and of the target word were synched with the monitor's vertical blank, there was a small amount of variability in the onsets of these events. Pilot testing revealed that this variability was sufficient to obscure priming effects. Response time outliers shorter than $100 \mathrm{~ms}$ and longer that $2,000 \mathrm{~ms}$ were removed before analysis. These values were chosen based on analysis of the pilot data and on the observed distribution of response times. Trials were also discarded when the comprehension question was answered incorrectly. Accuracy was quite high- $98.7 \%$, on averageindicating that participants were reading and comprehending the sentences. Altogether, approximately $4 \%$ of the trials were discarded.

Perea and Lupker (2003b) used ANOVAs to compare each related-prime condition to its matched unrelated control condition. That is, the researchers compared the identity prime condition to the unrelated-prime condition, and each TL condition to the matched SUB condition. These same comparisons were performed here by using contrast coding on the prime type variable to create a set of planned comparisons that compared each related prime (identity, TL internal, and TL final) to its unrelated-prime control (unrelated, SUB internal, and SUB final, respectively). The data were then analyzed using a linear mixed effects model (Baayen, Davidson, \& Bates, 2008). The model was fitted using a stepwise selection procedure, and the randomeffects structure was fitted using likelihood ratio tests. Random intercepts for participants and items were included, but no random slopes contributed to the model (all $p \mathrm{~s}>.99$ ). 
Only fixed effects and interactions that were significant (i.e., $p<.05)$ were retained in the model. The $p$ values were obtained using Markov chain Monte Carlo sampling. The response times were also log transformed.

The results of the mixed model analysis of response times are summarized in Table 2. The model revealed that the identity prime condition and the TL internal prime condition had significantly faster response times than did the unrelated and SUB internal prime control conditions, respectively. The reading times in the various prime conditions are represented in Fig. 2. The TL final prime condition did not differ from the SUB final control condition.

The results of Experiment 1 replicated the two primary findings of TL masked-priming research. First, the significant facilitative effect of TL internal priming indicated that letter identity can be extracted independently of letter position in a foveally presented word. Second, the lack of significant priming in the TL final condition reinforced the conclusion that not all letter positions were equal, such that the position encoding of internal letters was more flexible than that of final letters. ${ }^{1}$ Both results are consistent with earlier single-word identification studies (e.g., Chambers, 1979; Perea \& Lupker, 2003b).

In Experiment 1, we reused stimuli that had previously been presented foveally using masked priming (in Perea \& Lupker, 2003b, Exp. 1) as well as parafoveally (in Johnson et al., 2007, Exp. 1). The fact that we observed significant priming effects in our Experiment 1, along with the fact that these priming effects more closely match those observed by Perea and Lupker (2003b) in a masked-priming study with targets presented in isolation, confirms that the SPaM paradigm works as intended. Experiment 1 also revealed that embedding prime-target pairs in nonconstraining sentences does not significantly change the pattern of priming from that observed in isolated-word studies, at least for TL primes. Finally, the results of Experiment 1 seem to confirm that the difference between Perea and Lupker's (2003b) and Johnson et al.'s findings with regard to the effectiveness of TL final primes can most likely be attributed to differences in foveal versus parafoveal processing and not to other potential differences between the two studies.

On the basis of the success of Experiment 1, we suggest that SPaM is a useful synthesis, allowing for the presentation of masked primes during the (somewhat) normal reading of connected text. In the General Discussion, we will outline more fully the advantages of this new paradigm and argue that it is a viable alternative to both the boundary-

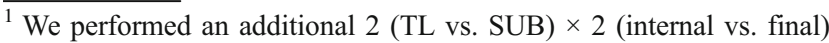
linear mixed effects model, which revealed a significant interaction of the two factors. This analysis confirmed that TL priming was observed only for internal letters.
}

Table 2 Summary of the fixed effects at the target word in Experiment 1

\begin{tabular}{lllll}
\hline Predictor & Coefficient & $S E$ & $t$ Value & $p$ \\
\hline Intercept & 5.7 & 0.05 & 114.46 & $<.001^{* * *}$ \\
$\begin{array}{l}\text { Identity vs. Unrelated } \\
\text { TL internal vs. SUB }\end{array}$ & -0.048 & 0.022 & -2.15 & $<.05^{*}$ \\
$\quad$ internal & -0.054 & 0.022 & -2.41 & $<.05^{*}$ \\
TL final vs. SUB final & -0.017 & 0.022 & 0.78 & .43 \\
\hline
\end{tabular}

${ }^{*} p \leq .05 .{ }^{* * *} p \leq .001$.

change paradigm (Johnson et al., 2007) and fast priming (Sereno \& Rayner, 1992) in eyetracking.

\section{Experiment 2}

In Experiment 1, we tested our new paradigm, a synthesis of masked priming and self-paced reading. Experiment 1 showed clearly that TL internal primes are facilitative in a nonconstraining context, but that word-final letters are privileged and that the transposition of word-final letters is no more facilitative than a matched substitution prime. This is the same pattern of results that was obtained in isolatedword experiments using the same materials (Perea \& Lupker, 2003b), indicating that our paradigm works much like traditional masked priming. In Experiment 2, we explored the effect of semantic predictability on TL effects by taking the target words from Experiment 1 and embedding them in highly constraining sentences.

Little is known about how semantic predictability should influence masked TL priming, but one thing is clear: Semantic predictability does not appear to reduce reliance on bottom-up input, but rather permits faster uptake of bottomup information, even when visual acuity is low. Although it is tempting to suppose that predictable words are not processed or are processed less carefully, this does not appear to be the case. The fact that words in highly constraining contexts are fixated for less time than unpredictable words, and are skipped more often, should not be taken as an indication that bottom-up visual information about the identity of these words is completely ignored or is unnecessary. A word that is skipped is still processed (Drieghe, Rayner, \& Pollatsek, 2005; Fisher \& Shebilske, 1985), and the evidence suggests that predictability increases word-skipping rates by making it easier to extract information from the parafovea, thereby reducing the need to fixate the word (Balota, Pollatsek \& Rayner, 1985). Also, when predictable words contain spelling errors, these errors still affect processing times, indicating that facilitation caused by predictability is not the result of less (or less careful) visual processing (Zola, 1984). Thus, it appears that predictability makes both parafoveal and foveal processing more efficient, 
Fig. 2 Reading times in Experiment 1. Error bars represent standard errors

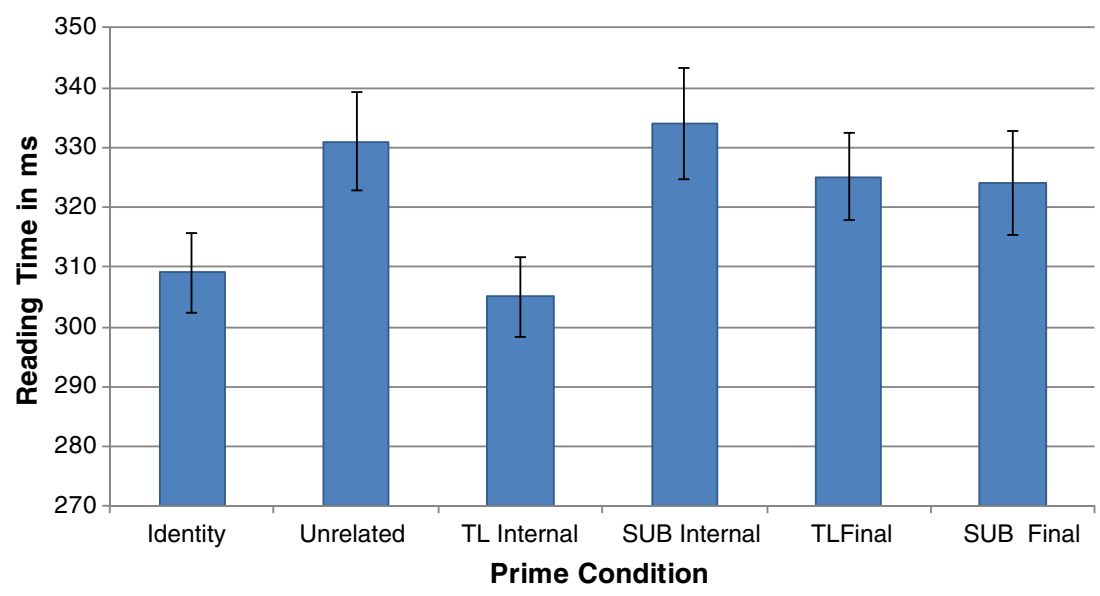

rather than reducing the need for visual input. Given this evidence, there is every reason to expect that the additional, bottom-up activation provided by masked primes should still be facilitative, even in highly constraining contexts. Less is known about how predictability affects reading behavior in the self-paced reading paradigm than in eyetracking studies, but clearly faster reading times should be expected. Since no parafoveal preview is available in selfpaced reading, the extraction of bottom-up information must occur during foveal processing. For this reason, and because a response is required after every word in self-paced reading, there are no grounds to expect "skipping" behavior in the experiments reported here.

Of course, because the constraining sentences used in Experiment 2 were expected to speed up reading times, it would not be surprising if the magnitude of observed priming effects was also affected. One possibility was that reading times would be reduced to the point that a floor effect would compress the means together and obscure any priming effects, even if they were or would otherwise be present. Another possibility was that the strong top-down activation from the context would reduce or eliminate priming effects by reducing reliance on bottom-up information, although, given the evidence cited above that predictability does not eliminate reliance on bottom-up information, this seemed unlikely. In either case, one would expect all prime conditions to be affected equally: If a floor effect or increased topdown activation reduced the apparent influence of bottomup factors, that influence should be reduced uniformly across all prime conditions, including the identity prime condition, effectively eliminating any priming effects.

On the other hand, if semantic predictability affects not just the magnitude of priming but the pattern of priming effects, there would seem to be two possible ways that this could occur. If the pattern of TL effects remained unchanged when the prime-target pairs were presented in these constraining sentences, this would indicate that semantic predictability does not influence the early process of letter position encoding. If the TL effect disappeared, however (i.e., the TL internal prime was not facilitative), this would indicate that semantic predictability has an extremely early influence on visual word recognition, and that readers predict both letter identity and letter position for highly constrained words.

A comparison between the patterns of priming observed in Experiments 1 and 2 would reveal the effect of predictability on the TL effect. Ideally, a comparison between TL priming in predictable and nonpredictable words would be made within participants in a single experiment, but there were several reasons to separate the predictable and nonpredictable sentences into two experiments. The primary reason was that TL priming requires several conditions, and including predictability as an additional factor would have made the experiment quite unwieldy, requiring many more participants and items. Additionally, SPaM is a new paradigm, and it was necessary to establish that traditional TL priming could be observed, as we did in Experiment 1. It was also unknown whether masked priming can occur for highly predictable stimuli, so it was necessary to test this, as we did in Experiment 2.

\section{Method}

Participants A group of 48 people from the University of Illinois at Urbana-Champaign community participated, the large majority of whom were recruited from the Educational Psychology subject pool. All were compensated for their time with either course credit or $\$ 5$, and none had participated in Experiment 1.

Materials One hundred twenty new sentences were created for the 120 target words used in Experiment 1. Each sentence was designed to make the target word as predictable as possible. Our goal was to achieve high cloze probabilities comparable to those of the medium- and high-contextualconstraint groups in Rayner and Well (1996). To assess our 
success, we recruited 28 participants (who did not participate in Exp. 1 or 2) to perform a sentence fragment completion task, in which they were given the first part of the sentence, up to but not including the target word, and asked to complete the sentence. The participants completed the sentence with the target word $75 \%$ of the time (range $=$ $13 \%-100 \%$ ), indicating that the sentences were highly constraining. This range of cloze probabilities matched the range of items that comprised Rayner and Well's medium- and high-constraint groups. The prime conditions in Experiment 2 were the same as those in Experiment 1. Additionally, 120 yes/no comprehension questions were created, one for each of the sentences.

Procedure The procedure was the same as in Experiment 1, with the exception that a question was asked after every trial. We included additional questions to encourage readers to read every sentence carefully, in spite of the predictable nature of the stimuli.

\section{Results and discussion}

Outliers and trials with incorrect comprehension question responses were trimmed in the manner described previously for Experiment 1. This resulted in the loss of $6.7 \%$ of the data. The question response accuracy was $94.5 \%$, indicating that participants were reading and understanding the sentences. As before, response times were log transformed and analyzed using a linear mixed model with the same planned comparisons described for Experiment 1. The target completion rates for each sentence were also included in the model, as a continuous predictor called "predictability." This was included to see whether response times and priming effects were influenced by the strength of the contextual constraint.

As mentioned above, there was a distinct possibility that no priming effects would be observed in Experiment 2 because the overall faster reading times for predictable words might create a floor effect, in which all of the means would be compressed together and no priming effects would be observable. For this reason, trial number was also included in the model as a fixed effect. It is commonly observed that participants speed up as they progress through a reading experiment, so it was possible that priming effects might appear in earlier trials, but disappear as the experiment progressed and participants' reading rates increased. If this were the case, the planned comparisons might interact with trial, such that priming effects would appear on earlier trials but disappear on later ones.

The results of the model are summarized in Table 3 . The $p$ values were obtained using Markov chain Monte Carlo sampling. Random intercepts for participants and items were included in the model, but no random slopes contributed to the fit (all $p \mathrm{~s}>.95$ ). The analysis revealed an interaction between prime type and trial. This interaction revealed a significant early identity-priming effect similar in magnitude to that observed in Experiment 1. This identity-priming effect shrunk as the experiment progressed and eventually disappeared, because participants sped up significantly in all non-identity-prime conditions over the course of the experiment. Thus, the disappearance of identity priming in later trials does appear to represent a floor effect in which response times were already so fast that no further facilitation was possible. This is supported by the fact that the mean reading time at the target words in Experiment 2 was $240 \mathrm{~ms}, 80 \mathrm{~ms}$ faster than the mean from Experiment $1(322 \mathrm{~ms})$. To confirm that there were no differences between the conditions in the second half of the experiment, the data set was divided, and each half of the experiment was analyzed separately. Consistent with the initial analysis, an identity-priming effect was observed for the first half of the experiment $(p<.05)$, but no TL effects were significant (both $t \mathrm{~s}<0.48$ ). Figure 3 represents the reading times in the different prime conditions observed in the first half of the experiment. No differences between prime conditions were observed in the second half of the experiment (all $t \mathrm{~s}<0.95$ ).

There are several reasons why the reading times in Experiment 2 might be so short, as compared to those in traditional masked priming studies and in Experiment 1. First, as participants were not required to make a lexical decision or to perform any task in addition to reading, it is unsurprising that reading times would be faster in the SPaM paradigm than in traditional masked-priming studies. Second, the target words in Experiment 2 appeared in highly constraining contexts, and it was expected that reading times would decrease as a result. If reading times had not turned out to be faster in Experiment 2 than in Experiment 1, this would have been cause for concern. Rayner and Well (1996) observed a 60-ms difference in total reading times between their high- and medium-constraint words and their low-constraint words, which is comparable to the 80 -ms difference between our Experiments 1 and 2. Third, as mentioned above, reading times were measured from the onset of the target word, so that participants had about $60 \mathrm{~ms}$ (10-ms pause $+50-\mathrm{ms}$ prime) to plan a buttonpress. When the upcoming word was highly constrained, it is likely that participants began preparing the buttonpress earlier, as they could be quite confident in their ability to quickly recognize the target word. All of the sentences in Experiment 2 involved highly predictable words, and participants never encountered any unpredictable or unexpected words. Under such circumstances, it is understandable that participants would adjust their reading strategy and speed up. Even though reading times at the target word were quite fast, the question response 
Table 3 Summary of the fixed effects at the target word in Experiment 2

${ }^{*} p \leq .05 .{ }^{* * *} p \leq .001 .{ }^{ \pm} p \leq .10$.

\begin{tabular}{lllll}
\hline Predictor & Coefficient & $S E$ & $t$ Value & $p$ \\
\hline Intercept & 5.42 & 0.0355 & 152.72 & $<.001^{* * *}$ \\
Identity vs. Unrelated & -0.063 & 0.029 & -2.19 & $<.05^{*}$ \\
TL internal vs. SUB internal & 0.0047 & 0.029 & 0.16 & .87 \\
TL final vs. SUB final & -0.00015 & 0.028 & -0.01 & .99 \\
Trial & -0.00074 & 0.00012 & -6.13 & $<.001^{* * *}$ \\
Predictability & 0.055 & 0.03 & -1.84 & $.066^{+}$ \\
Interaction: Identity vs. Unrelated $\times$Trial & 0.00086 & 0.00042 & 2.07 & $<.05^{*}$ \\
Interaction: TL internal vs. SUB internal $\times$ Trial & 0.00016 & 0.00042 & 0.37 & .71 \\
Interaction: TL final vs. SUB final $\times$ Trial & -0.000084 & 0.00041 & -0.20 & .83 \\
\hline
\end{tabular}

accuracy was still very high, indicating that participants understood what they had read.

The gradual disappearance of the identity-priming effect that was observed here is consistent with intuition, theory, and previous research, which all predict faster reading times and less observable influence for bottom-up factors with increased response speed. However, the significant identity-priming effect early in the experiment shows that bottom-up factors continued to play a role, although that role might be difficult to observe in behavioral measures when response times were particularly fast.

Figure 3 shows no effect of TL internal priming, contrary to what was observed in Experiment 1. Neither the TL internal prime nor the TL final prime condition differed from their respective control conditions in Experiment 2 in either half of the experiment. The fact that identity priming was observed in both Experiments 1 and 2, while TL priming occurred only in the nonconstraining sentences of Experiment 1, indicated that semantic predictability eliminates the TL effect; that is, letter position encoding is less flexible in more constraining contexts. The presence of an identity-priming effect in Experiment 2 made it clear that the absence of TL priming could not be attributed to a general weakening of bottom-up effects as a result of strong top-down influence; in that case, predict- ability should have reduced the effectiveness of all primes, including the identity prime, and not just the TL internal prime. Likewise, the disappearance of TL priming could not be attributed to a floor effect that "squashed" the means together and masked TL effects, for two reasons. First, in Experiment 1 the TL-priming effect was numerically equivalent to the identity-priming effect, so both priming effects should have been equally affected and equally reduced. Second, the interaction of prime type and trial indicated that reading times did not reach floor until later trials, so if a TL effect was present, it should have been observable in the earlier trials, just as the identity-priming effect was. Even if there was a "hidden" TL effect in Experiment 2 that we were not able to detect, the fact that we were able to detect the identity-priming effect in both experiments indicated that context modulated the effect of the TL prime but not of the identity prime. If context did not have an effect on TL priming, no difference between Experiments 1 and 2 would have been observed. As the patterns of TL priming differed between the two experiments, predictability did have an effect on TL priming.

Table 3 also shows that there was a marginal effect of predictability, indicating that as contextual constraint increased, reading times decreased. It is perhaps unsurprising
Fig. 3 Reading times in the first half of Experiment 2. Error bars represent standard errors

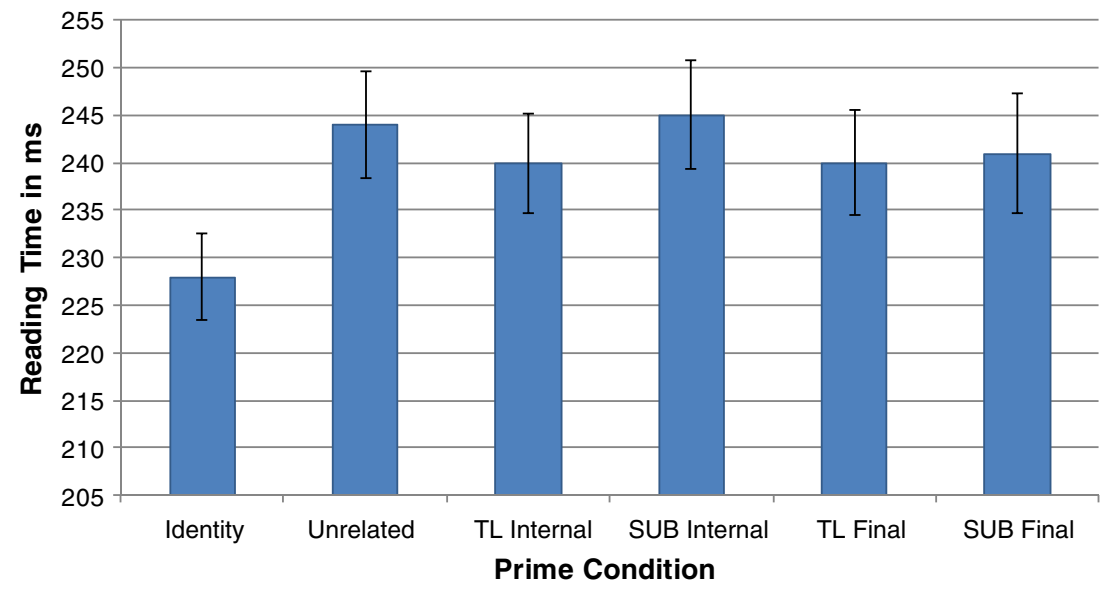


that the effect of predictability was only marginally significant, given that all of the items were highly constrained by context. Because predictability did not interact with either prime type or trial (all $t \mathrm{~s}<0.84$ ), the final best-fitting model did not include these interactions.

To more directly compare the results of Experiments 1 and 2, we performed an analysis on the combined data from Experiment 1 and from the first half of Experiment 2. The fixed effects included the same planned comparisons described in Experiment 1 for prime condition, as well as the effect of experiment (1 vs. 2). Random effects included random intercepts for participants and items and random by-participant slopes for the prime condition contrasts. There was an overall effect of experiment (coeff. $=-0.29$, $S E=0.06, t=-4.77, p<.001$ ), with shorter reading times in Experiment 2. The model revealed significant priming in the identity (coeff. $=-0.024, S E=0.0096, t=-2.48$, $p<.005$ ) and TL internal (coeff. $=-0.027, S E=0.011$, $t=-2.51, p<.001)$ conditions, but not in the TL final condition $(t<1.18)$. The TL internal contrast interacted with experiment (coeff. $=0.03, S E=0.014, t=2.19, p<.005$ ), confirming that TL priming disappeared in Experiment 2. The identity contrast did not interact with experiment $(t<$ $1.13)$, indicating that the magnitudes of identity priming were comparable across the two experiments.

In sum, identity priming, but not TL priming, was observed in Experiment 2, while both identity priming and TL priming (for internal transpositions) were observed in Experiment 1. The implications of these findings will be discussed in more detail below.

\section{General discussion}

We will first discuss what the results of Experiment 1 reveal about the foveal and parafoveal processing of visually presented words. Second, we will discuss what the experiments reported here reveal about prediction during reading. Third, we will explore the implications of these experiments for models of visual word recognition. Finally, we will discuss the potential uses of the SPaM paradigm from Experiments 1 and 2 and the advantages it offers over traditional masked priming and other paradigms.

TL effects in the fovea and the parafovea

Overall, the results of Experiment 1 are more consistent with the masked-priming data of Perea and Lupker (2003b) than with Johnson et al.'s (2007) parafoveal preview data. Perea and Lupker (2003b) reported a greater effect of the TL manipulation in the TL internal condition than in the TL final condition, and this same difference in priming was observed in our Experiment 1. Johnson et al., on the other hand, observed equal priming for both TL conditions when the prime was presented in the parafovea. Taken together, these three studies lead to several conclusions. First, letter identity information can be, generally speaking, extracted independently of letter position information, as demonstrated in previous lexical decision and naming studies (Chambers, 1979; Christianson et al., 2005; Perea \& Lupker, 2003b). Second, letter-encoding flexibility is not restricted to words presented in isolation; the TL effect applies to words in (nonconstraining) contexts as well. Third, in the fovea, the position of the external letters (specifically, the final letter) is important; transposing the final letters is just as disruptive as substituting them with other letters. Fourth, in the parafovea, the position of the final letter is less important, at least for the fiveletter words examined here and by Johnson et al. Those authors speculated that the parafoveal insensitivity to the word-final letter position was due to parafoveal eccentricity: The farther from the fovea a given letter is, the less information will be available for processing, including whether or not that letter is an edge (word-final letter). The results obtained in Experiment 1 here support this speculation: When we presented the words from Perea and Lupker's (2003b) study in the self-paced masked-priming paradigm used here, in the same sentences in which Johnson et al. had presented those words, the results replicated those of Perea and Lupker (2003b).

\section{Prediction during reading}

A variety of experimental methods have revealed that semantic predictability facilitates word recognition (Federmeier \& Kutas, 1999; Fischler \& Bloom, 1979; Rayner \& Well, 1996). Some researchers have argued that this facilitation occurs after lexical access (Stanovich \& West, 1983), while others have proposed that highly specific predictions generated online can simplify the process of visual word recognition itself (DeLong et al., 2005; Laszlo \& Federmeier, 2009). The primary goal of the experiments reported here was to investigate whether readers make predictions about upcoming words, and how detailed those predictions are.

Our findings indicate that letter position encoding becomes less flexible in predictable words. That is, it appears that a highly constraining context can prompt readers to predict the identity of upcoming words. Importantly, this prediction appears to be more specific than simply a prediction of general semantic meaning, or even of the lemma of the predicted word. Rather, it seems that the basic visual features of the word - down to letter identity and position-are predicted. In cases in which either the identity or position (or both) does not appear as predicted, processing difficulty arises. Because letter position encoding appears to become less flexible in the later stages of visual word recognition (White et al., 2008), this finding 
also suggests that predictions made online during reading can influence visual word recognition from its earliest stages.

Of course, the level of constraint needed to eliminate the TL effect in Experiment 2 was quite high, with target words having an average cloze score of .75. For the nonconstraining sentences used in Experiment 1, the TL effect remained strong. Thus, Experiment 1 indicates that under normal circumstances readers may still generate predictions, but the influence of these weaker predictions does not permeate the earlier stages of visual word recognition. Future research examining this issue should examine the relationship between the degree of contextual constraint and prediction strength. Additionally, a within-participants manipulation of predictability would provide even stronger evidence for prediction and would be a useful follow-up to the present study.

Implications for models of visual word recognition

As we discussed in the introduction, current models of visual word recognition explain TL effects in mostly bottom-up terms, with some concessions made to take into account the influence of certain lexical factors. The results reported here clearly indicate that bottom-up factors are not the whole story when visual word recognition is considered within the larger context of reading connected text. The fact that semantic predictability and other top-down influences have been neglected is not surprising, as visual word recognition research has been conducted almost exclusively on words presented in isolation.

We do not mean to suggest that it is wrong to study words in isolation or to explain TL effects in bottom-up terms; the results of Experiment 1 reveal that under most circumstances, such methods and explanations are more than sufficient to describe the phenomena of interest. Rather, we echo the suggestion of Rayner and Reichle (2010) that "future efforts to understand the cognitive processes and representations involved in identifying words during reading might benefit from a more careful consideration of how lexical processing both constrains and is constrained by the other components of reading" (p. 790). Research and theory on visual word recognition processes are sufficiently advanced that it is not only possible, but necessary, to study words in context. Such research would help to adjudicate between competing theories by answering important questions in the field, such as how independent the word recognition system is from other language comprehension (and production) processes. It would also help to bridge the gap between theories of visual word recognition and theories of eye movement control in reading, such as the E-Z Reader model (Reichle, Pollatsek, Fisher, \&
Rayner, 1998; Reichle, Warren, \& McConnell, 2009), which assume that eye movements are controlled, at least in part, by word recognition processes. Clearly, more research on word recognition in connected text will be needed before these goals can be accomplished.

Advantages and uses of the self-paced reading with masked priming (SPaM) paradigm

The combination of self-paced reading and masked priming described above provides one unique contribution to existing masked-priming paradigms: Because the primetarget pair is embedded in a sentence, this paradigm makes it possible to explore top-down influences on visual word recognition, as we did in Experiment 2. Although more research is clearly needed to understand and perfect the SPaM technique, the results of Experiment 1 show that the priming effects produced by our new paradigm should generally be similar to those observed in isolated-word studies, barring any influence of context, of course.

The possibilities of this paradigm have prompted other researchers to combine masked priming with either selfpaced reading (Trueswell \& Kim, 1998) or eyetracking (Sereno \& Rayner, 1992). The technique described here has one significant advantage over both of these previous implementations: The primes are not so obvious. Trueswell and Kim reported that $12 \%$ of their participants were able to identify more than half of the primes. The obviousness of the primes forced Trueswell and Kim to explicitly instruct participants to ignore the primes, and this overt mention of the primes probably led some participants to search for them (see Trueswell \& Kim, 1998). Similarly, Sereno and Rayner reported that many of their participants were aware of the presence of a prime, although the participants were only able to identify the prime $10 \%-20 \%$ of the time. In both studies, only one of the words in the sentence was primed, so the flash caused by the prime was quite noticeable. By contrast, while a few participants in the present experiments were aware of a "flash" before each word, only one of our participants reported noticing the presence of the primes, and none were able to identify any of the primes, as determined in postsession interviews in which the presence of the primes was first divulged to participants. Presenting a prime before every word in the sentence made the individual primes less noticeable, but not less effective. Another advantage of this approach is that, since each word in the sentence is already preceded by a prime, it would be quite easy to include more than one prime-target pair per sentence. A third advantage is that in the SPaM paradigm the forward mask is hash marks (\#), as is common in maskedpriming studies, instead of underlines, as in Trueswell and Kim's study. 
Priming every word in the sentence is possible with selfpaced reading but would be impractical for eyetracking, because of the high rate of data loss. When combined with masked primes, the temporal and spatial sensitivity that for many tasks makes eyetracking preferable to self-paced reading becomes a liability. Sereno and Rayner (1992) reported that a great deal of their data were unusable. For example, sometimes the display change was triggered prematurely, and sometimes participants skipped over the target word altogether. Approximately 14\% of Sereno and Rayner's participants did not provide enough usable data (less than $60 \%$ ) and had to be discarded. For those participants who remained, an average of $26 \%$ of the data were unusable. By contrast, only between $4 \%$ and $10 \%$ of the trials in our experiments were discarded, and all participants provided usable data. If Sereno and Rayner had attempted to prime more than one word per sentence, the percentage of data lost would have been prohibitively high. We note that the main reason for discarding data in the present experiments was due to participants incorrectly answering comprehension questions. A lower number of questions would therefore result in even fewer data being lost.

When working with certain limited populations, losing the data of even 1 participant is unfortunate. Researchers working with aphasics, second language learners, or small populations of native speakers often cannot afford to write off even a few participants because of data loss. In such cases, the self-paced reading plus masked priming paradigm described here would be preferable to fast priming with an eyetracker.

SPaM has one final advantage over fast priming: economy. Fast priming requires an eyetracker, whereas the present paradigm does not. This means that researchers who currently do masked priming with isolated words can run experiments with primes in sentences on the same hardware that they currently use, rather than upgrading to an eyetracker.

\section{Conclusion}

Using a novel paradigm that combines self-paced reading and masked priming, we explored the effect of semantic predictability on the transposed-letter effect. In nonconstraining sentences, we observed considerable flexibility in letter position encoding for internal letters, but not for external ones, consistent with previous research. In highly constraining sentences, however, letter position encoding was universally inflexible. This finding suggests that in constraining contexts readers make highly specific predictions about the identity and position of the letters in upcoming words, and that these predictions have an early influence on visual word recognition.
Author Note The authors thank Jeong-Ah Shin, Jung Hyun Lim, Hee Youn Cho, Anastasia Gusicova, Cassie Landry, Kacey Wochna, and the rest of the members of the Educational Psychology Psycholinguistics Lab for assistance in running participants. This research was funded in part by a grant from the Campus Research Board at UIUC, by an Arnold O. Beckman Award to K.C., and by generous support from the Department of Educational Psychology, UIUC.

\section{References}

Aaronson, D., \& Ferres, S. (1984). The word-by-word reading paradigm: An experimental and theoretical approach. In D. E. Kieras \& M. A. Just (Eds.), New methods in reading comprehension research (pp. 29-68). Hillsdale, NJ: Erlbaum.

Aaronson, D., \& Scarborough, H. S. (1976). Performance theories for sentence coding: Some quantitative evidence. Journal of Experimental Psychology. Human Perception and Performance, 2, 5670. doi:10.1037/0096-1523.2.1.56

Acha, J., \& Perea, M. (2008a). The effect of neighborhood frequency in reading: Evidence with transposed-letter neighbors. Cognition, 108, 290-300. doi:10.1016/j.cognition.2008.02.006

Acha, J., \& Perea, M. (2008b). The effects of length and transposedletter similarity in lexical decision: Evidence with beginning, intermediate, and adult readers. British Journal of Psychology, 99, 245-264. doi:10.1348/000712607X224478

Altmann, G. T. M., \& Kamide, Y. (1999). Incremental interpretation at verbs: Restricting the domain of subsequent reference. Cognition, 73, 247-264. doi:10.1016/S0010-0277(99)00059-1

Andrews, S. (1996). Lexical retrieval and selection processes: Effects of transposed-letter confusability. Journal of Memory and Language, 35, 775-800. doi:10.1006/jmla.1996.0040

Baayen, R. H., Davidson, D. J., \& Bates, D. M. (2008). Mixed-effects modeling with crossed random effects for subjects and items. Journal of Memory and Language, 59, 390-412. doi:10.1016/j. jm1.2007.12.005

Balota, D. A., Pollatsek, A., \& Rayner, K. (1985). The interaction of contextual constraints and parafoveal visual information in reading. Cognitive Psychology, 17, 364-390. doi:10.1016/0010-0285 (85)90013-1

Chambers, S. M. (1979). Letter and order information in lexical access. Journal of Verbal Learning and Verbal Behavior, 18, 225-241. doi:10.1016/S0022-5371(79)90136-1

Christianson, K., Johnson, R. L., \& Rayner, K. (2005). Letter transpositions within and across morphemes. Journal of Experimental Psychology: Learning, Memory, and Cognition, 31, 1327-1339. doi:10.1037/0278-7393.31.6.1327

Davis, C. J. (1999). The self-organizing lexical acquisition and recognition (SOLAR) model of visual word recognition. Unpublished doctoral dissertation, University of New South Wales.

Davis, C. J. (2010). The spatial coding model of visual word identification. Psychological Review, 117, 713-758. doi:10.1037/ a 0019738

DeLong, K. A., Urbach, T. P., \& Kutas, M. (2005). Probabilistic word pre-activation during language comprehension inferred from electrical brain activity. Nature Neuroscience, 8, 1117-1121. doi: $10.1038 / \mathrm{nn} 1504$

Drieghe, D., Rayner, K., \& Pollatsek, A. (2005). Eye movements and word skipping during reading revisited. Journal of Experimental Psychology. Human Perception and Performance, 31, 954-969. doi:10.1037/0096-1523.31.5.954

Duffy, S. A., Henderson, J. M., \& Morris, R. K. (1989). The semantic facilitation of lexical access during sentence processing. Journal of Experimental Psychology: Learning, Memory, and Cognition, 15, 791-801. doi:10.1037/0278-7393.15.5.791 
Duñabeitia, J. A., Perea, M., \& Carreiras, M. (2007). Do transposedletter similarity effects occur at a morpheme level? Evidence for morpho-orthographic decomposition. Cognition, 105, 691-703. doi:10.1016/j.cognition.2006.12.001

Duñabeitia, J. A., Perea, M., \& Carreiras, M. (2009). There is no clam with coats in the calm coast: Delimiting the transposed-letter priming effect. Quarterly Journal of Experimental Psychology, 62, 1930-1947. doi:10.1080/17470210802696070

Ehrlich, S. F., \& Rayner, K. (1981). Contextual effects on word perception and eye movements during reading. Journal of Verbal Learning and Verbal Behavior, 20, 641-655. doi:10.1016/S00225371(81)90220-6

Federmeier, K. D. (2007). Thinking ahead: The role and roots of prediction in language comprehension. Psychophysiology, 44, 491-505. doi:10.1111/j.1469-8986.2007.00531.x

Federmeier, K. D., \& Kutas, M. (1999). A rose by any other name: Long-term memory structure and sentence processing. Journal of Memory and Language, 41, 469-495. doi:10.1006/ jmla.1999.2660

Fischler, I., \& Bloom, P. A. (1979). Automatic and attentional processes in the effects of sentence contexts on word recognition. Journal of Verbal Learning and Verbal Behavior, 18, 1-20. doi:10.1016/ S0022-5371(79)90534-6

Fisher, D. F., \& Shebilske, W. L. (1985). There is more that meets the eye than the eye-mind assumption. In R. Groner, G. W. McConkie, \& C. Menz (Eds.), Eye movements and human information processing (pp. 149-158). Amsterdam: Elsevier.

Forster, K. I. (1989). Basic issues in lexical processing. In W. D. Marsen-Wilson (Ed.), Lexical representation and process (pp. 75-107). Cambridge, MA: MIT Press.

Forster, K. I., Davis, C., Schoknecht, C., \& Carter, R. (1987). Masked priming with graphemically related forms: Repetition or partial activation? Quarterly Journal of Experimental Psychology, 39A, 211-251. doi:10.1080/14640748708401785

Forster, K. I., Mohan, K., \& Hector, J. (2003). The mechanics of masked priming. In S. Kinoshita \& S. J. Lupker (Eds.), Masked priming: State of the art (pp. 3-37). Hove, UK: Psychology Press.

Frisson, S., Rayner, K., \& Pickering, M. J. (2005). Effects of contextual predictability and transitional probability on eye movements during reading. Journal of Experimental Psychology: Learning, Memory, and Cognition, 31, 862-877. doi:10.1037/02787393.31.5.862

Gomez, P., Ratcliff, R., \& Perea, M. (2008). The overlap model: A model of letter position coding. Psychological Review, 115, 577600. doi:10.1037/a0012667

Grainger, J., \& Whitney, C. (2004). Does the huamn mnid raed wrods as a wlohe? Trends in Cognitive Sciences, 8, 58-59. doi:10.1016/ j.tics.2003.11.006

Hess, D. J., Foss, D. J., \& Carroll, P. (1995). Effects of global and local context on lexical processing during language comprehension. Journal of Experimental Psychology. General, 124, 62-82. doi:10.1037/0096-3445.124.1.62

Holmes, V. M., \& Ng, E. (1993). Word-specific knowledge, wordrecognition strategies, and spelling ability. Journal of Memory and Language, 32, 230-257. doi:10.1006/jmla.1993.1013

Johnson, R. L., Perea, M., \& Rayner, K. (2007). Transposed-letter effects in reading: Evidence from eye movements and parafoveal preview. Journal of Experimental Psychology. Human Perception and Performance, 33, 209-229. doi:10.1037/0096-1523.33.1.209

Jordan, T. R., \& Thomas, S. M. (2002). In search of perceptual influences of sentence context on word recognition. Journal of Experimental Psychology: Learning, Memory, and Cognition, 28, 34-45. doi:10.1037/0278-7393.28.1.34

Just, M. A., Carpenter, P. A., \& Woolley, J. D. (1982). Paradigms and processes in reading comprehension. Journal of Experimental Psychology. General, 111, 228-238. doi:10.1037/0096-3445.111.2.228
Kamide, Y., Altmann, G. T. M., \& Haywood, S. L. (2003). The timecourse of prediction in incremental sentence processing: Evidence from anticipatory eye movements. Journal of Memory and Language, 49, 133-156. doi:10.1016/S0749-596X(03)00023-8

Kliegl, R., Grabner, E., Rolfs, M., \& Engbert, R. (2004). Length, frequency, and predictability effects of words on eye movements in reading. European Journal of Cognitive Psychology, 16, 262284. doi:10.1080/09541440340000213

Ktori, M., \& Pitchford, N. J. (2008). Effect of orthographic transparency on letter position encoding: A comparison of Greek and English monoscriptal and biscriptal readers. Language \& Cognitive Processes, 23, 258-281. doi:10.1080/01690960701536797

Kutas, M., \& Hillyard, S. A. (1984). Brain potentials during reading reflect word expectancy and semantic association. Nature, 307, 161-163. doi:10.1038/307161a0

Laszlo, S., \& Federmeier, K. D. (2009). A beautiful day in the neighborhood: An event-related potential study of lexical relationships and prediction in context. Journal of Memory and Language, 61, 326-338. doi:10.1016/j.jml.2009.06.004

Lau, E., Almeida, D., Hines, P. C., \& Poeppel, D. (2009). A lexical basis for N400 context effects: Evidence from MEG. Brain and Language, 111, 161-172. doi:10.1016/j.bandl.2009.08.007

Lee, C. H., \& Taft, M. (2009). Are onsets and codas important in processing letter position? A comparison of TL effects in English and Korean. Journal of Memory and Language, 60, 530-542. doi:10.1016/j.jml.2009.01.002

Lupker, S. J., Perea, M., \& Davis, C. J. (2008). Transposed-letter effects: Consonants, vowels and letter frequency. Language \& Cognitive Processes, 23, 93-116. doi:10.1080/01690960701579714

Marslen-Wilson, W. D. (1989). Access and integration: Projecting sound onto meaning. In W. D. Marslen-Wilson (Ed.), Lexical representation and process (pp. 3-24). Cambridge, MA: MIT Press.

Morris, R. K. (1994). Lexical and message-level sentence context effects on fixation times in reading. Journal of Experimental Psychology: Learning, Memory, and Cognition, 20, 92-103. doi:10.1037/0278-7393.20.1.92

Norris, D. (1994). Shortlist: A connectionist model of continuous speech recognition. Cognition, 52, 189-234. doi:10.1016/00100277(94)90043-4

Norris, D., Kinoshita, S., \& van Casteren, M. (2010). A stimulus sampling theory of letter identity and order. Journal of Memory and Language, 62, 254-271. doi:10.1016/j.jml.2009.11.002

O'Connor, R. E., \& Forster, K. I. (1981). Criterion bias and search sequence bias in word recognition. Memory \& Cognition, 9, 7892. doi:10.3758/BF03196953

Otten, M., Nieuwland, M. S., \& van Berkum, J. J. (2007). Great expectations: Specific lexical anticipation influences the processing of spoken language. BMC Neuroscience, 8,89 . doi:10.1186/ 1471-2202-8-89

Perea, M., \& Carreiras, M. (2008). Do orthotactics and phonology constrain the transposed-letter effect? Language \& Cognitive Processes, 23, 69-92. doi:10.1080/01690960701578146

Perea, M., \& Lupker, S. J. (2003a). Does jugde activate COURT? Transposed-letter similarity effects in masked associative priming. Memory \& Cognition, 31, 829-841. doi:10.3758/BF03196438

Perea, M., \& Lupker, S. J. (2003b). Transposed-letter confusability effects in masked form priming. In S. Kinoshita \& S. J. Lupker (Eds.), Masked priming: State of the art (pp. 97-120). Hove, U. K.: Psychology Press.

Perea, M., \& Lupker, S. J. (2004). Can CANISO activate CASINO? Transposed-letter similarity effects with nonadjacent letter positions. Journal of Memory and Language, 51, 231-246. doi:10.1016/j.jml.2004.05.005

Rayner, K., \& Reichle, E. D. (2010). Models of the reading process. Wiley Interdisciplinary Reviews: Cognitive Science, 1, 787-799. doi:10.1002/wcs.68 
Rayner, K., \& Well, A. D. (1996). Effects of contextual constraint on eye movements in reading: A further examination. Psychonomic Bulletin \& Review, 3, 504-509. doi:10.3758/BF03214555

Rayner, K., White, S. J., Johnson, R. L., \& Liversedge, S. P. (2006). Raeding wrods with jubmled lettres: There is a cost. Psychological Science, 17, 192-193. doi:10.1111/j.1467-9280.2006.01684.x

Reichle, E. D., Pollatsek, A., Fisher, D. L., \& Rayner, K. (1998). Toward a model of eye movement control in reading. Psychological Review, 105, 125-157. doi:10.1037/0033-295X.105.1.125

Reichle, E. D., Warren, T., \& McConnell, K. (2009). Using E-Z Reader to model the effects of higher level language processing on eye movements during reading. Psychonomic Bulletin \& Review, 16, 1-21. doi:10.3758/PBR.16.1.1

Rueckl, J. G., \& Rimzhim, A. (2011). On the interaction of letter transpositions and morphemic boundaries. Language and Cognitive Processes, 26, 482-508.

Schneider, W., Eschman, A., \& Zuccolotto, A. (2002). E-Prime user's guide. Pittsburgh, PA: Psychology Software Tools.

Schoonbaert, S., \& Grainger, J. (2004). Letter position coding in printed word perception: Effects of repeated and transposed letters. Language \& Cognitive Processes, 19, 333-367.

Sereno, S. C., \& Rayner, K. (1992). Fast priming during eye fixations in reading. Journal of Experimental Psychology. Human Perception and Performance, 18, 173-184. doi:10.1037/0096-1523.18.1.173

Stanovich, K. E., \& West, R. F. (1979). Mechanisms of sentence context effects in reading: Automatic activation and conscious attention. Memory \& Cognition, 7, 77-85. doi:10.3758/BF03197588

Stanovich, K. E., \& West, R. F. (1983). On priming by a sentence context. Journal of Experimental Psychology. General, 112, 1-36. doi:10.1037/0096-3445.112.1.1

Trueswell, J. C., \& Kim, A. E. (1998). How to prune a garden path by nipping it in the bud: Fast priming of verb argument structure. Journal of Memory and Language, 39, 102-123. doi:10.1006/ jmla.1998.2565

van Berkum, J. J. A., Brown, C. M., \& Hagoort, P. (1999). Early referential context effects in sentence processing: Evidence from event-related brain potentials. Journal of Memory and Language, 41, 147-182. doi:10.1006/jmla.1999.2641

van Berkum, J. J. A., Brown, C. M., Zwitserlood, P., Kooijman, V., \& Hagoort, P. (2005). Anticipating upcoming words in discourse: Evidence from ERPs and reading times. Journal of Experimental Psychology: Learning, Memory, and Cognition, 31, 443-467. doi:10.1037/0278-7393.31.3.443

White, S. J., Johnson, R. L., Liversedge, S. P., \& Rayner, K. (2008). Eye movements when reading transposed text: The importance of word-beginning letters. Journal of Experimental Psychology. Human Perception and Performance, 34, 1261-1276. doi:10.1037/ 0096-1523.34.5.1261

Whitney, C. (2001a). How the brain encodes the order of letters in a printed word: The SERIOL model and selective literature review. Psychonomic Bulletin \& Review, 8, 221-243. doi:10.3758/BF03196158

Whitney, C. (2001b). Position-specific effects within the SERIOL framework of letter-position coding. Connection Science, 13, 235-255. doi:10.1080/09540090110083659

Wicha, N. Y. Y., Bates, E. A., Moreno, E. M., \& Kutas, M. (2003). Potato not Pope: Human brain potentials to gender expectation and agreement in Spanish spoken sentences. Neuroscience Letters, 346, 165-168. doi:10.1016/S0304-3940(03)00599-8

Wicha, N. Y. Y., Moreno, E. M., \& Kutas, M. (2003). Expecting gender: An event related brain potential study on the role of grammatical gender in comprehending a line drawing within a written sentence in Spanish. Cortex, 39, 483-508. doi:10.1016/ S0010-9452(08)70260-0

Wicha, N. Y. Y., Moreno, E. M., \& Kutas, M. (2004). Anticipating words and their gender: An event-related brain potential study of semantic integration, gender expectancy, and gender agreement in Spanish sentence reading. Journal of Cognitive Neuroscience, 16, 1272-1288. doi:10.1162/0898929041920487

Zola, D. (1984). Redundancy and word perception during reading. Perception \& Psychophysics, 36, 277-284. doi:10.3758/ BF03206369 